\title{
Insulinoma misdiagnosed as epilepsy ${ }^{*}$
}

\author{
Ouadii Mouaqit , Ayman Boubouh, Lahcen Ifrine, El Hadj Omar El Malki, Raouf Mohsine, \\ Abdelkader Belkouchi
}

Surgery Departement A, Ibn Sina University Hospital, Rabat, Morocco

Email: \#mouaqit1975@hotmail.com

Received 17 November 2011; revised 4 January 2012; accepted 13 January 2012

\begin{abstract}
Insulinoma is a rare disease presenting with episodic neuroglycopenic and/or adrenergic symptoms. We describe the clinical case of a female subject, 44 years old, affected with insulinoma, misdiagnosed for two years as epilepsy, threaten with antiepileptics. Insulinoma was diagnosed based on fasting blood glucose level of $15 \mathrm{mg} / \mathrm{dl}$, high fasting immunoreactive insulin/blood glucose ratio (more than 0.3 ), and a tumor in the pancreas by abdominal CT. After surgical removal of the neoplasm, the blood glucose level and insulin level turned normal. A benign insulinoma was also confirmed by histopathological evaluation. The patient remained seizure-free during the 2-year follow up.
\end{abstract}

Keywords: Insulinoma; Epilepsy; Convulsions; Hypoglycemia; Surgery

\section{INTRODUCTION}

Insulinoma is a very rare tumor with a reported incidence of 0.5 to 5 patients/million [1-3]. It is caused by overproduction of insulin due to neoplastic proliferation of pancreatic islet $\beta$ cells. It can secrete insulin in short bursts and cause fluctuation of blood glucose level correspondingly, and then the patients will have intermittent neuroglycopenic symptoms, such as conscious disorder, abnormal behavior, psychiatric symptoms or convulsion. Epileptic seizures can be mimicked by many conditions including hypoglycemia. The authors report on a patient with hypoglycemic spells caused by insulinoma misdiagnosed as intractable epilepsy.

\section{CASE PRESENTATION}

A 44 years old woman, Mrs. J who was apparently all right 2 years back, when she started having generalized weakness, recurrent convulsions, confusions, hypogly-

\footnotetext{
${ }^{*}$ The author(s) declare that they have no competing interests.

\#Corresponding author.
}

cemic episodes during fasting, and weight gain of $10 \mathrm{~kg}$. She had been diagnosed with epilepsy by a general practitioner and antiepileptic treatment had been prescribed. The frequency of attacks had increased up to 3 times a week in the previous 4 months. She was referred to endocrinology division after discovering repeated low levels of glycemia $<45 \mathrm{mg} / \mathrm{dl}$. She appeared good, suffering with important sweating and recurrent tonic-clonic attacks, There was no associated tongue bite or history of head injury. Each episode was associated with unconsciousness lasting from few minutes to a few hours. BP: $120 / 60$, the heamo-gluco-test showed $36 \mathrm{mg} / \mathrm{dl}$, she was treated with hypertonic glucose solution with resolution of symptoms. Laboratory tests pointed out altered insulin (basal insulin $51 \mathrm{uU} / \mathrm{ml})$ and C-peptide $(7 \mathrm{ug} / \mathrm{ml})$ dosages, as well as glycaemic levels which resulted low especially in night hours and early morning. Ct-scan of the abdomen showed the presence of a nodular image in pancreatic tail that was suspicious for insulinoma with no lymph node implication and without other secondary localizations; MR confirmed this finding by showing a tissular and cystic lesion in the pancreatic tail (Figure 1).

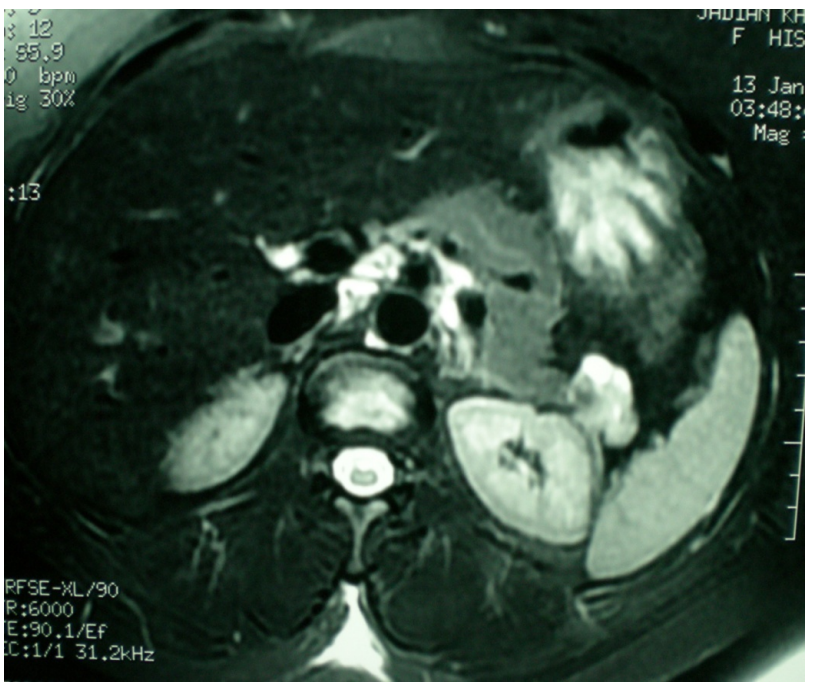

Figure 1. Axial T1 weighted fast spin echo image show the lesion in the neck of the pancreas to have solid and cystic components. 
The presence of MEN syndrome was excluded. Subject was submitted to surgical excision of insulinoma (Figure 2). After surgical removal of the neoplasm, the blood glucose level and insulin level turned normal. A benign insulinoma was also confirmed by histopathological. The patient remained seizure-free during the 2-year follow up.

\section{DISCUSSION}

A common problem faced by general practitioners and hospital doctors alike is the elderly patient with hypoglycaemia. Such a patient may present atypically with confusion, behavioural changes. More serious conesquences such as seizures or falls with fractures may result. That hypoglycaemia might be a cause may be ignored. Insulinoma is very rare. Insulinoma may occur in either sex at any age. The majority of insulinomas are sporadic, usually small and benign (>90\%) and occur throughout the pancreas; rarely they are ectopic [3]. The interval from presentation to diagnosis ranged from 1 month to 30 years (median 2 years) [3]. The delay in its diagnosis is caused by several factors. Firstly, the symptoms of insulinoma lack specificity, including various seizure disorders, personality change, bizarre behavior, amnesia, convulsions, and incidentally dystonia and polyneuropathy; these symptoms are similar with many common neurological and psychiatric disorders. Secondly, fasting blood glucose level can be normal in some patients. Thirdly, hypoglycemia itself is able to decrease the counterregulatory hormonal responses to hypoglycemia and induce unawareness of autonomic and neuroglycopenic symptoms $[4,5]$. The diagnosis of insulinoma is often delayed as there may be a myriad of symptoms associated with neuroglycopoenia, including neurologycal features as confusion who is the most common, motor deficits, convulsion, blurred vision, coma and an adrenergic response which apparently, due to the rarity of the insulinoma, seem to be strictly related to the tumor itself [6]. Unless there is the presence of localized abnormalities in the cerebral circulation, low glucose level affects all cerebral neurons, resulting in a generalized dysfunction which may present as diffuse slow activity in EEG and lead the clinician to prescribe antiepileptics. The episodic nature of hypoglycemia. in insulinoma also causes the symptoms to fluctuate, and delays the diagnosis. An international investigation of 1067 cases showed that neuropsychiatric symptoms including loss of consciousness, sluggishness, confusion, asthenia, deep coma, dizziness, disturbance in vision and epilepsy are present in $92 \%$ of the subjects [7]. In our subject the clinical presentation that was suggestive of epilepsy caused a 2 year diagnostic delay. She had been treated for two years as epilepsy. It is reported that before the confirmation of diagnosis of insulinoma, $39 \%$ of the cases are diagnosed

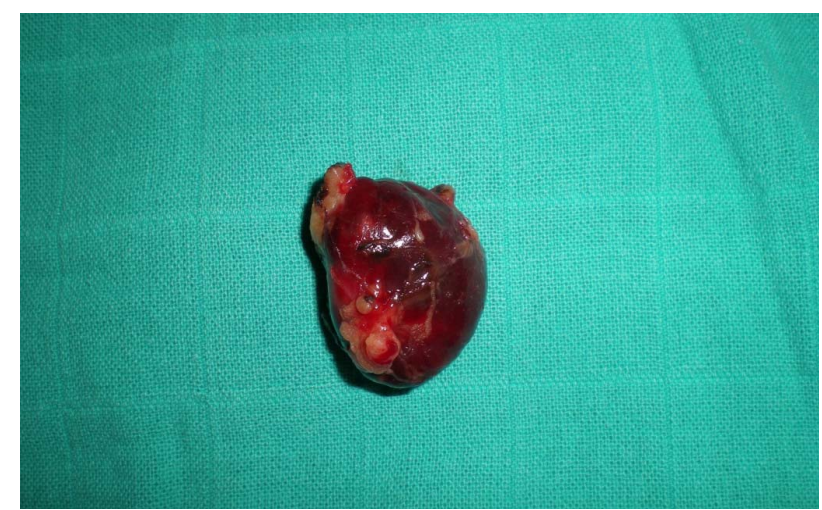

Figure 2. Macroscopy of the enucleated nodule.

as epilepsy, $12 \%$ of the cases are treated with antiepileptic drugs, and $89 \%$ of patients with insulinoma are confused while $64 \%$ of them have personality changes and abnormal behaviors [8]. Many subjects report an association between symptoms and meals or activity and stress; others report the symptoms during night time. In malignnant tumors, the postprandial episodes are frequent [9]. In our subject there was a weight gain of $10 \mathrm{~kg}$; this symptom is aspecific and is reported in only $20 \%$ of the subjects [10]. However, this symptom must not be under-estimated especially if it is due to "therapeutic" ingestion of foods. Biochemical tests are the mainstay of diagnosis: the demonstration of high insulin levels in the case of hypoglycaemia; C-peptide assay to exclude factitious hypoglycaemia, as low or absent C-peptide levels suggest exogenous insulin administration; the anti-insulin antibodies frequently present in those subjects are the most frequently used test. Once the diagnosis of insulinoma has been bio chemically confirmed, the issue of whether localizing techniques are of benefit arises. In fact, the assumption that preoperative localization of insulinoma is of benefit at all is still unproven [11]. Other authors believe it necessary to give the surgeon information about the tumor and the strategies to plan the operation. Endoscopic sonography and MRI are useful in preoperative diagnosis of small pancreatic insulinomas [12]. In several experienced centers endoscopic sonography is the initial and often the only preoperative imaging test performed. Conventional CT scan is usually unhelpful; however, multiphase thin slice enhanced CT greatly increases the detection rate, and small insulinomas can be readily found in arterial phase image. Since simple intraoperative palpation of the pancreas detects $75 \%-95 \%$ of insulinomas and the more experienced surgeons report only $0,5 \%$ tumors missed on palpation, we believe that preoperative localization is useful but not indispensable [13]. Once the diagnosis of insulinoma has been reached and the tumor eventually localized, the most common form of treatment is surgical which is either curative or useful in obtaining a good biochemical palliation. 


\section{CONCLUSION}

Insulinoma, though uncommon, it's a potentially recognizable and treatable disease, as long as there is a high index of suspicion. It should always be considered among the diagnostic possibilities in any patient with unusual or inexplicable neurological features, including atypical convulsions refractory to pharmacotherapy.

\section{REFERENCES}

[1] Ding, Y., Wang, S., Liu, J., Yang, Y., Liu, Z., Li, J., Zhang, B., Chen, Y. and Ding, M. (2010) Neuropsychiatric profiles of patients with insulinomas. European $\mathrm{Neu}$ rology, 63, 48-51.

[2] Crespel, A., Barbaud, A., Andrieu, J.M., Oziole, E., Coubes, P. and Gélisse, P. (2009) Insulinoma presenting as pseudo-drug-resistant focal epilepsy. Revue Neurologique, 165, 493-495. doi:10.1016/j.neurol.2008.08.004

[3] Gnacńska, M., Lewczuk and A., Sworczak, K. (2008) Insulinoma misdiagnosed and treated as epilepsy. Polski Merkuriusz Lekarski, 24, 251-253.

[4] Wang, S., Hu, H.T., Wen, S.Q., Wang, Z.J., Zhang, B.R. and Ding, M.P. (2008) An insulinoma with clinical and electroencephalographic features resembling complex partial seizures. Journal of Zhejiang University Science B, 9, 496-499. doi:10.1631/jzus.B0720011

[5] Blasetti, A., Di Pietro, L., di Corcia, G., Franzoni, E., Chiarelli, F. and Verrotti, A. (2007) Can insulinoma cause generalised epilepsy? Journal of Pediatric Endocrinology and Metabolism, 20, 837-840. doi:10.1515/JPEM.2007.20.7.837

[6] Suzuki, K., Miyamoto, M., Miyamoto, T. and Hirata, K.
(2007) Insulinoma with early-morning abnormal behavior. Internal Medicine, 46, 405-408.

doi:10.2169/internalmedicine.46.6071

[7] Alemdar, M., Iseri, P. and Komsuoglu, S.S. (2006) Insulinoma in differential diagnosis of seizure disorder. Journal of Neuropsychiatry and Clinical Neuroscience, 18, 247-248. doi:10.1176/appi.neuropsych.18.2.247-a

[8] Striano, P. and Striano, S. (2006) Insulinoma presenting as refractory late-onset epilepsy. Epilepsia, 47, 452. doi:10.1111/j.1528-1167.2006.00444_6.X

[9] Twardowschy, C.A., Leite, S.A., Outi, T.Y. and Dykyj, M.T. (2005) Insulinoma presenting as seizure: Case report. Arquivos de Neuro-Psiquiatria, 63, 685-688. doi:10.1590/S0004-282X2005000400026

[10] Graves, T.D., Gandhi, S., Smith, S.J., Sisodiya, S.M. and Conway, G.S. (2004) Misdiagnosis of seizures: Insulinoma presenting as adult-onset seizure disorder. Journal of Neurol Neurosurg Psychiatry, 75, 1091-1092 doi:10.1136/jnnp.2003.029249

[11] Muscatiello, N., Cuomo, R., Gentile, M., De Francesco, V., Stoppino, V., Contillo, A., Salcuni, A., Natale, C., Panella, C. and Ierardi, E. (2003) Endoscopic ultrasound localization of a solitary insulinoma of pancreatic tail misdiagnosed as epilepsy: Case report. Abdominal Imaging, 28, 859-861. doi:10.1007/s00261-003-0039-5

[12] Striano, S., Striano, P., Manganelli, F., Boccella, P., Bruno, R., Santoro, L. and Percopo, V. (2003) Distal hypoglycemic neuropathy. An insulinoma-associated case, misdiagnosed as temporal lobe epilepsy. Neurophysiologie Clinique, 33, 223-227. doi:10.1016/j.neucli.2003.08.001

[13] Schwaninger, M., Haehnel, S., Hess, K., et al. (2002) Cerebellar ataxia after repeated hypoglycemia. European Journal of Neurology, 9, 541-545. doi:10.1046/j.1468-1331.2002.04454.x 